\title{
NIÑAS TRABAJADORAS EN CIUDAD DE MÉXICO. APROXIMACIONES Y REINTERPRETACIONES CONCEPTUALES ${ }^{1}$
}

\author{
Working girls in Mexico City. Conceptual approaches and reinterpretations
}

BEGOÑA LEYRA FATOU ${ }^{2}$

\section{Resumen}

El trabajo infantil femenino sigue siendo, hoy por hoy, un gran desconocido, debido a que se ha subsumido dentro del universal masculino de los niños trabajadores, sin tener en cuenta las particularidades y especificidades de las niñas. Partiendo de premisas de la antropología urbana, del trabajo y de género, a través de este artículo, pretendo analizar los límites que se establecen entre la ciudad como espacio masculinizado y adulto y las niñas que trabajan en las calles de la Ciudad de México, mostrando algunas de las estrategias que ellas configuran y que les sirven de amortiguación a los envites que los entornos laborales y vitales les producen, reconstruyendo, de algún modo, su percepción de la ciudad convirtiéndola en un espacio menos dañino, a pesar de la complejidad estructural que les rodea.

Este artículo, ante todo, es una crítica a los discursos y a los programas de atención social e intervención, que sin tener en cuenta la propia percepción de las niñas, toman decisiones sobre la vida de estas con el fin de «proteger» y que, sin embargo, subordinan y enclaustran más que favorecen su propia autonomía y empoderamiento. Así mismo, esta aproximación pretende desmitificar algunas premisas que rodean a esta práctica y visibilizar a las niñas en el contexto social mexicano, dándole a esta realidad la complejidad que se merece evitando caer en las generalizaciones simplistas de los discursos «oficiales».

Palabras clave: Trabajo Infantil, Niñas trabajadoras, Hábitat Urbano, Desigualdad de género, Dicotomía público/privado.
Abstract

Currently, there is little research on female child labour, most of the time it has been subsumed within the masculine universe of male child labour, without considering the specific particularities of girls. Based on assumptions of urban anthropology, gender and work, the limits that are established between the masculinized and adult city area and the female children who work in the streets of Mexico City are analyzed in this article showing some of the strategies that the girls shape. These strategies serve to buffer the stakes that the working and living environments produce for them, reconstructing, somehow, their perception of the city making it a less dangerous area, despite the structural complexity that surrounds them.

This article, first of all, is a critique of discourses and social care programs and intervention, which regardless of one's perception of girls, make decisions about their lives in order to "protect» them however, instead they subordinate and cloister the girls rather than favor their own autonomy and empowerment. Moreover, this approximation intends to demystify some principles around this practice and to look at the girls in the Mexican social context, showing the complexity that this matter deserves in an attempt to avoid the simplistic generalizations of the «official» version.

Keywords: Child Labour, female child labourers, Urban Habitat, Gender inequality, public/private dichotomy.

Recibido: 22/01/2012 Revisado: 12/03/2012 Aceptado: 04/05/2012 Publicado: 02/11/2012

1. El presente artículo es una pequeña síntesis y reflexión a partir de la tesis doctoral en antropología presentada por la misma autora en 2009 en la Universidad Complutense de Madrid y que se encuentra en el repositorio de la UCM: http://eprints.ucm.es/9394/1/T31016.pdf

2. Begoña Leyra Fatou. Universidad Complutense de Madrid. Departamento de Trabajo Social y Servicios Sociales. Escuela Universitaria de Trabajo Social. Despacho 2113. Campus de Somosaguas. 28223 Pozuelo de Alarcón. Madrid. Teléfono y fax: +34913942730 / +34913942722 E-mail: mbleyra@trs.ucm.es 


\section{Introducción}

El siguiente artículo es fruto de las observaciones y reflexiones obtenidas gracias al trabajo de campo etnográfico realizado en México durante dos años, como parte de mi doctorado en Antropología Social y que configuró la base de mi tesis doctoral sobre las niñas trabajadoras en Ciudad de México (Leyra, 2009), sobre la que a día de hoy sigo investigando y reflexionando.

Partiendo de premisas de la antropología urbana, del trabajo y de género, pretendo analizar los límites que se establecen entre la ciudad como espacio masculinizado y adulto y las niñas trabajadoras en la calle, mostrando algunas de las estrategias que ellas configuran y que les sirven de amortiguación a los envites que los entornos laborales y vitales les producen, reconstruyendo, de algún modo, su percepción de la ciudad convirtiéndola en un espacio menos dañino, a pesar de la complejidad estructural que les rodea.

Desde un enfoque teórico, vemos cómo las personas encargadas de la planificación urbana, suelen trabajar con categorías abstractas tales como «población» «hogares», «familias» y en consecuencia, las respuestas que se dan en esta materia no contemplan la heterogeneidad de necesidades del universo social, contribuyendo a reproducir las discriminaciones de género. Los estudios sobre el hábitat urbano (Falú, Morey y Rainero, 2002: 19), entendido como una construcción histórico-social, han conducido necesariamente a intersecciones entre distintas disciplinas y campos de conocimiento. La categoría de análisis de género en estos estudios constituye una herramienta básica para analizar cómo se expresan las desigualdades y diferencias en la vivencia del espacio urbano y de qué manera el espacio contribuye a reproducir las relaciones de género en cada contexto geográfico, social y temporal específico.

Tradicionalmente, los mencionados estudios y la propia configuración y construcción social de hombres y mujeres y niños y niñas, han producido una relegación de las mujeres a la esfera de lo privado, considerando el ámbito público y productivo de los varones. El movimiento feminista ${ }^{3}$, durante varias décadas ha tratado (y en parte, ha logrado) de derrumbar esas dicotomías excluyentes promoviendo la igualdad entre hombres y mujeres, y replanteando una nueva manera de enfocar las relaciones de poder. A través de las diferentes «olas del feminismo», se han producido grandes reivindicaciones como terminar con el patriarcado, reivindicar lo personal como político, denunciar la opresión sobre las mujeres, mejorar los derechos sexuales y reproductivos

3. Sería interminable citar todas las reflexiones y reivindicaciones teóricas aportadas desde el feminismo, pudiendo servir de referencia las obras de Beauvoir, Wollstonecraft, Friedan y Pateman, entre muchas otras (ver referencias completas en la bibliografía). 
con legislación sobre anticoncepción e interrupción voluntaria del embarazo, o la búsqueda de la igualdad en el plano profesional y/o educativo. Dichas reivindicaciones, hoy en día, y a pesar de los muchos avances, siguen siendo factor clave en las agendas políticas de y para las mujeres y las niñas.

Partiendo de las aportaciones críticas de movimientos feministas se comienzan a cuestionar los principios universales de los estudios y se trata de salvar la invisibilidad de la mujer, invisibilidad especialmente reseñable en el ámbito urbano, ya que los estudios que contemplaban el paradigma de lo general, consideraban lo universal como masculino y lo particular como femenino, cayendo una vez más en las peligrosas dicotomías construidas alrededor de las relaciones entre los géneros ${ }^{4}$.

En el caso de las niñas, al igual que ocurre con los estudios de las mujeres, estos se engloban dentro del masculino, ignorando, silenciando o dando por obvias las especificidades que tienen ellas en los fenómenos o situaciones que se estudian, dejando de lado aspectos susceptibles de reflexión antropológica y de intervención social como es la participación de las niñas en esferas públicas, el trabajo como elemento de socialización y como parte de las relaciones familiares, el uso del tiempo, del espacio o del dinero, el control social en proporción diferenciada a las niñas que a los niños, sin tener en cuenta las diferencias de género, perpetuando las desigualdades legitimadas por el peso de la tradición y de la cultura.

La infancia, además, está en muchas ocasiones en el punto de mira de la población adulta y la búsqueda de su bienestar da lugar a múltiples actividades organizadas por esta, bien sea de forma particular o bien estructuradas por las diversas instituciones públicas y entidades privadas, cuyo interés se centra en proporcionar una mayor calidad de sus condiciones de vida. La mayoría de las actividades programadas para el bienestar de la infancia y adolescencia, están guiadas por el principio de su «mejor interés» tal y como este es interpretado desde el sector adulto. Aunque, lamentablemente, siguen siendo limitadas las ocasiones en las que dicha población tiene la oportunidad de expresar su propia idea respecto a sus intereses, o puede participar en la toma

4. Sobre las dicotomías en función de los géneros, está el modelo androcéntrico que plantea los roles del hombre como activo, fuerte, pragmático y con dominio de la razón, mientras que las mujeres destacan en sus roles de pasividad, debilidad, afectividad y sentimiento. Frente a este modelo son interesantes las aportaciones que han realizado desde la antropología Michelle Z. Rosaldo (sobre la dicotomía Doméstico y Público) y Sherry B. Ortner (sobre la dicotomía de Naturaleza y Cultura) (ver referencias completas en la bibliografía). 
de decisiones de los asuntos que directamente les conciernen (Gaitán y Martínez, 2006: 7-8).

La Convención sobre los Derechos del Niño (CDN) de las Naciones Unidas, incorporada al ordenamiento jurídico de los países que la han suscrito, introduce, junto a los derechos de protección y provisión, derechos de participación de los niños y niñas en la vida social; su desarrollo favorece el cambio de su papel de receptores pasivos de la benevolencia adulta, por el de personas responsables de sí mismas, que contribuyen activamente a la construcción del bien común. Los caminos para alcanzar el objetivo de la plena integración de los niños, las niñas y los adolescentes en la vida social son diversos, y entre ellos se encuentran las actividades organizadas en forma de planes, programas o proyectos de intervención, desarrollados ampliamente por las instituciones de carácter educativo, lúdico, promocional o social.

La introducción de formas de participación de las y los propios actores interesados en los procesos de planificación que tienen como destinataria a la población infantil y adolescente, así como de una visión transversal del reconocimiento y respeto de los derechos de los niños y niñas en dichos procesos, puede contribuir de forma muy positiva no sólo al mayor acierto en sus medidas y a la mejor consecución de buenos resultados, sino también a una más amplia y profunda consideración de las personas menores de edad como miembros de la sociedad y como sujetos de derechos.

En suma, existen al menos, tres razones, que justifican la necesidad y conveniencia de aplicar el enfoque de derechos en la consideración de la infancia y la adolescencia (Liebel, 2006: 23):

- La posición de los niños y niñas en la sociedad: débil, subordinada al criterio y al juicio de la población adulta, basada en una falta de confianza en su capacidad, lo cual inhibe su progresiva aceptación y afrontamiento de responsabilidades.

- La posición de la parte adulta: entre la protección y la indiferencia, alentada por visiones nostálgicas y estereotipadas de la infancia, lo que dificulta el desarrollo de la autonomía personal y de la capacidad de hacer de los niños y niñas.

- La Convención sobre los Derechos del Niño, que reconoce derechos de carácter vinculante y señala caminos para que las opiniones, experiencias y aspiraciones de las personas menores de edad puedan expresarse y sean tomadas en cuenta.

El concepto fundamental de la Convención sobre los Derechos del Niño es el llamado interés superior del niño y comprende al niño y a la niña no sólo como 
objeto de medidas de protección y asistencia sino como titular de derechos propios y como sujeto de su propia vida y desarrollo, y como tal es capaz y debe participar en las determinaciones que le conciernen.

De igual modo, para hacer frente a este estudio, tal y como veremos en el siguiente apartado, es preciso aclarar y revisar el propio concepto de trabajo infantil (desde diferentes enfoques y posicionamientos políticos) para ver el punto de partida y evitar sesgos en las interpretaciones del fenómeno. Es importante aclarar, además, que todos los conceptos suponen un punto de partida pero no son el único marco de interpretación y análisis, ya que la realidad siempre es mucho más compleja y habrá que ahondar en ella a través de la aproximación etnográfica. El trabajo infantil ha sido y es objeto de diversas interpretaciones y ha sido profusamente abordado desde las ciencias sociales (Bolton, Pole y Mizen 2001; Dorman 2001; Woodhead, 2004; Aitken, Estrada, Jennings y Aguirre, 2006; Hungerland, Liebel, Milne y Wihstutz, 2007; Guerra, Calderón y Soto, 2007; López-Calva, 2007; Diallo, Hagemann, Etienne, Gurbuzer y Mehran, 2011) y para este estudio fue necesario revisar pormenorizadamente todas las aportaciones para poder situar el caso particular de las niñas mexicanas.

\section{Revisando los conceptos}

El trabajo, al igual que otros acontecimientos que implican a mujeres y niñas, ha sido contemplado dentro de los estudios científicos como parte de un todo, sin hacer incidencia en las especificidades que estas tienen como colectivo diferenciado de los hombres y los niños. Asimismo, la propia definición de trabajo ha recogido numerosas acepciones donde no se ven claramente las fronteras que lo delimitan, y no siempre se han tenido en consideración de análisis aquellos elementos que evidenciaban las importantes aportaciones laborales de las mujeres en las sociedades (no sólo dentro del ámbito estrictamente familiar y/o reproductivo $)^{5}$.

Desde una perspectiva general, se puede definir trabajo como «la aportación específicamente humana que conjuntamente con los recursos naturales permite obtener los bienes y servicios necesarios para la satisfacción de las necesidades humanas» (Carrasco, 1996).

Desde una perspectiva antropológica, podemos definir trabajo como «el término que designa las diversas formas inventadas por el hombre para actuar

5. La antropología de género se ha preocupado de analizar esta situación en profundidad, son muchas las aportaciones teóricas, una de las publicaciones clásicas sería el libro de Martín y Voorhies (1978). 
sobre su entorno natural y extraer de él los medios materiales de su existencia social» (Bonte e Izard, 1996: 707).

Vemos que ambas definiciones pueden servir a nivel general pero habrá que concretarlas en cada sociedad y especificar cuáles son las relaciones sociales que lo determinan. El trabajo, además, no está exento de las relaciones entre hombres y mujeres, ya que los sistemas de género son un factor estructurante de la división del trabajo, siendo uno de sus factores constitutivos, y no meramente el resultado que deriva de tal división.

Así mismo, cada vez es más frecuente, que desde diversas disciplinas se critique la ambigüedad y la falta de precisión del concepto «trabajo» sobre todo cuando este es aplicado a las actividades que realizan mujeres y niñas. Las críticas se forjan en torno al carácter sesgado de esta categoría, haciendo énfasis en la necesidad de analizar las distintas formas que posee la actividad laboral, tanto remunerada como no remunerada. Especialmente equiparando los términos de «trabajo» y «empleo», ya que tradicionalmente el término trabajo se ha utilizado de manera más amplia (abarcando remuneración o no remuneración), mientras que el término empleo se refiere de manera específica, al trabajo mercantil remunerado (Carrasco, Mayordomo, Domínguez y Alabart, 2004).

\subsection{Trabajo Infantil}

Según el Fondo de las Naciones Unidas para la Infancia (UNICEF), el concepto de trabajo infantil puede ser contemplado desde diferentes niveles (en Alarcón, 1994: 135): Un primer nivel que considera al trabajo infantil como toda actividad que realizan los niños y niñas en el campo de la producción, comercialización y servicios, incluyendo todas las ocupaciones realizadas en el sector informal, en las empresas formales, en el campo, además de las tareas domésticas y el ejercicio de la prostitución. En esta aproximación, la mendicidad y el robo, a pesar de ser conductas de subsistencia, permanecen al margen del sistema económico, pudiéndose utilizar para ellas la categoría planteada por UNICEF de «actividades marginales de ingreso».

Un segundo nivel, de carácter más restrictivo, define trabajo infantil como toda actividad lícita que realizada por niños y niñas tenga las siguientes características:

- Participación directa en procesos de producción, comercialización o prestación de servicios.

- Dichos bienes o servicios han de ser consumidos principalmente fuera del hogar del niño o la niña. 
- Por tales actividades se puede recibir o no una retribución, la cual no es necesariamente en dinero.

- Dicha participación supone regularidad temporal, según ciertas horas al día o días a la semana.

A nivel general, UNICEF (1997: 32-45) ${ }^{6}$ plantea que son muchas las modalidades de trabajo infantil en todo el mundo, pero estas pueden agruparse en siete tipos principales:

- Trabajo doméstico

- Trabajo servil o forzoso

- La explotación sexual con fines comerciales

- Trabajo en la industria y en las plantaciones

- Trabajo en la calle

- Trabajo para la familia

- Trabajo de las niñas (contemplado como caso específico debido a que es un sector de la población con una problemática añadida que va desde el acoso sexual hasta la exclusión de la educación).

Sin embargo, hay otros planteamientos teóricos (Liebel, 2003: 60) que prefieren no limitar la definición a aspectos estadísticos, a valoraciones morales o a términos económicos o hacer equivalente el término a actividades concretas, ni siquiera a calificar de trabajo infantil aquello que está dentro del «entender común» ya que este no existe a nivel mundial, y abogan más por una definición que tenga en cuenta los supuestos específicos de cada cultura, entendiendo de manera amplia el mayor número de actividades que resulten objetivas y/o subjetivas para la reproducción individual y social, considerando un amplio espectro de formas de trabajo (aunque pueda parecer una definición demasiado amplia) que sirva para diferenciarla de otras actividades infantiles sin olvidar el amplio espectro de formas de trabajo, caracterizadas culturalmente, incluyendo valoraciones subjetivas y asignaciones de significado.

\subsection{Explotación Laboral Infantil}

El trabajo infantil es un concepto que se emplea como término genérico para referirse a los trabajos que realizan los niños y las niñas y que no tienen necesariamente consecuencias negativas para estos. UNICEF (1997: 24) además,

6. Cada año UNICEF publica su «Estado Mundial de la Infancia», y en 1997 presentó su monográfico sobre Trabajo Infantil, siendo hoy en día una referencia clásica en relación a esta temática. 
reconoce que existen una gran variedad de actividades cuyo desempeño no implica un efecto negativo en el desarrollo de estos niños y niñas. El trabajo infantil pasa a ser explotación laboral infantil cuando las condiciones en las que se encuentran estos niños y niñas dificultan su acceso a la escuela, cuando conllevan un peligro en su realización o son de algún modo perjudiciales para su bienestar físico, mental, moral o social. UNICEF explica que para que el trabajo infantil sea explotación, se deben dar las siguientes características:

- Trabajo a tiempo completo a una edad demasiado temprana

- Horario laboral prolongado

- Trabajos que producen tensiones indebidas de carácter físico, social o psicológico

- Trabajo y vida en la calle en malas condiciones

- Remuneración inadecuada

- Demasiada responsabilidad

- Trabajos que obstaculizan el acceso a la educación

- Trabajos que socavan la dignidad y autoestima de los niños y niñas, tales como la esclavitud o el trabajo servil y la explotación sexual

- Trabajos que perjudican el pleno desarrollo social y psicológico.

La Organización Internacional del Trabajo (OIT), en 1999, adoptó el Convenio $\mathrm{N}^{\circ} 182$ sobre las Peores Formas del Trabajo Infantil, en la Conferencia Internacional del Trabajo en Ginebra, compuesta por delegados de las organizaciones de empleadores, sindicatos y gobiernos de los 175 países que eran Estados miembros de la Organización Internacional del Trabajo. Las actividades que son consideradas «peores formas» en dicho convenio serían las siguientes (Hilowitz, 2004: 44-60):

- Esclavitud o prácticas análogas, como la venta y el tráfico de niños, servidumbre por deudas y trabajo forzoso u obligatorio (incluido el reclutamiento forzoso de niños para utilizarlos en conflictos armados);

- Utilización u oferta de niños para la prostitución o para la pornografía;

- Utilización u oferta de niños para actividades ilícitas, como la producción y el tráfico de estupefacientes;

- Trabajo que por su naturaleza o por las condiciones en que se lleva a cabo es probable que dañe la salud, seguridad o moralidad de los niños, esto es, «trabajo peligroso».

Por tanto, habrá que revisar (para evitar confusiones e identificaciones de ambos conceptos) tanto en la consideración sobre el trabajo infantil como en la consideración de la explotación laboral infantil, diferentes variables que lo 
configuran como un fenómeno complejo, que va más allá del aspecto económico y que forma parte del bagaje cultural, de la experiencia vital de sucesivas generaciones y como parte del conjunto familiar, es decir, debe ser analizado como un fenómeno multidimensional (Estrada, 1999a: 179). La infancia trabajadora es un fenómeno que a lo largo del mundo y desde tiempos pasados se repite, tanto en los países en desarrollo como en los países desarrollados. Bajo nuevas estimaciones de la OIT (Diallo, Hagemann, Etienne, Gurbuzer y Mehran, 2011), los datos aportan que en 2008 había aproximadamente 306 millones de niños y niñas económicamente activos de cinco a 17 años de edad, 215 millones de los cuales podrían considerarse niñas y niños trabajadores. De estos últimos, 115 millones realizaban trabajos peligrosos (74 millones de niños y 41 millones de niñas), pero estas cifras no incluyen a aquellos (especialmente a millones de niñas) que trabajan dentro del ámbito del hogar y que no reciben salario alguno.

\section{Metodología}

Este artículo es una pequeña parte de las observaciones y reflexiones obtenidas gracias al trabajo de campo etnográfico realizado, en secuencia longitudinal, desde septiembre de 2002 a septiembre de 2005 (en dos fases de trabajo de campo con un total de 21 meses), con niñas trabajadoras y sus entornos familiares en Ciudad de México.

Para llevar a cabo esta investigación, se hizo especial énfasis en las técnicas propias de la Antropología Social y Cultural (de la etnografía clásica u holística) y de las técnicas cualitativas de las Ciencias Sociales en general. Las técnicas utilizadas han sido: entrevistas individuales en profundidad (semi-estructuradas); entrevistas informales (no estructuradas); observación participante en varios espacios comunes a las niñas y niños, así como de sus familias y comunidades; acompañamiento en las rutinas laborales, apoyo en las tareas escolares, acompañamiento en actividades lúdicas; historias de vida de sus familiares y genealogías laborales, con el fin de buscar aspectos de la socialización que rodean al trabajo infantil; revisión de fuentes bibliográficas, hemerográficas y documentales; consulta de fuentes estadísticas e indicadores sociales; y análisis de dibujos (combinados durante las entrevistas a las niñas y niños más pequeños).

La producción científica sobre etnografía con infancia ha sido apenas desarrollada y ocasionó al principio algunas dificultades, teniendo que desplegar otras estrategias de aproximación al sujeto de estudio, adaptando las técnicas señaladas a las diferentes condicionantes que rodeaban cada caso, por ejemplo, combinando técnicas de entrevista con dibujos (que arrojaban 
mucha información y eran una manera de romper la monotonía que puede significar una entrevista al uso para una niña de 9 años), o hacer juegos con la grabadora antes de las entrevistas (rompiendo la distancia que esta genera en las entrevistas). Las entrevistas llegaron tras mucho tiempo de aproximación y siempre tras un trabajo previo con las familias. De igual modo, fue crucial para el trabajo la observación participante, que arrojó mucha más información que las propias entrevistas.

Tabla 1: Resumen del Trabajo de Campo

\begin{tabular}{|l|c|c|c|}
\hline \multicolumn{1}{|c|}{ INFORMANTES } & $\begin{array}{c}1^{\text {a }} \text { FASE DE } \\
\text { TRABAJO DE } \\
\text { CAMPO (10 MESES) }\end{array}$ & $\begin{array}{c}2^{\text {a }} \text { FASE DE } \\
\text { TRABAJO DE } \\
\text { CAMPO (11 } \\
\text { MESES) }\end{array}$ & $\begin{array}{c}\text { TOTALES (21 } \\
\text { MESES) }\end{array}$ \\
\hline $\begin{array}{l}\text { Unidades } \\
\text { Domésticas (U.D.) }\end{array}$ & 14 & 15 & 29 \\
\hline Niñas & 13 & 16 & 29 \\
\hline Niños & 6 & 2 & 17 \\
\hline Mujeres & 3 & 3 & 61 \\
\hline Hombres & 30 & 51 & 6 \\
\hline $\begin{array}{l}\text { Entrevistas } \\
\text { realizadas }\end{array}$ & & & \\
\hline
\end{tabular}

Fuente: Elaboración propia

Para llevar a cabo el trabajo de campo fueron elegidos siete lugares de observación: Barrio de Tepito, Colonia Morelos y Colonia Santa María La Ribera (situadas en la Delegación Cuauhtémoc), Mercado de Sonora y Mercado de La Merced (situados en la Delegación Venustiano Carranza), Central de Abasto de la Ciudad de México (situado en la Delegación Iztapalapa) y Basurero de Chimalhuacán (situado en el Municipio conurbado de Chimalhuacán), que contenían una gran representación de tipologías laborales infantiles siendo además zonas con gran actividad comercial de la Ciudad de México, fueron seleccionadas 29 niñas (y sus respectivas unidades domésticas, elegidas en función de variables como el tipo de trabajo desempeñado por la niña, el tipo de unidad doméstica a la que pertenecía -teniendo en cuenta los criterios de residencia y parentesco-, el lugar de trabajo y la edad), con edades comprendidas entre los 4 y 15 años (en México la celebración de los 15 años es un rito que supone una frontera entre la infancia y la adolescencia), que trabajan 
en la calle $e^{7}$ e en espacios públicos (solas o acompañadas), dentro del ámbito urbano (específicamente en la Zona Metropolitana de Ciudad de México). Dentro del ámbito de observación y de análisis han estado las propias niñas trabajadoras, sus familias, así como también algunos niños trabajadores que permitieron analizar las diferencias de género, realizando un total de 81 entrevistas en profundidad.

\section{Mapa 1: Zona metropolitana de ciudad de México \\ (Delegaciones y Municipios Conurbados)}

\section{LEYENDA}
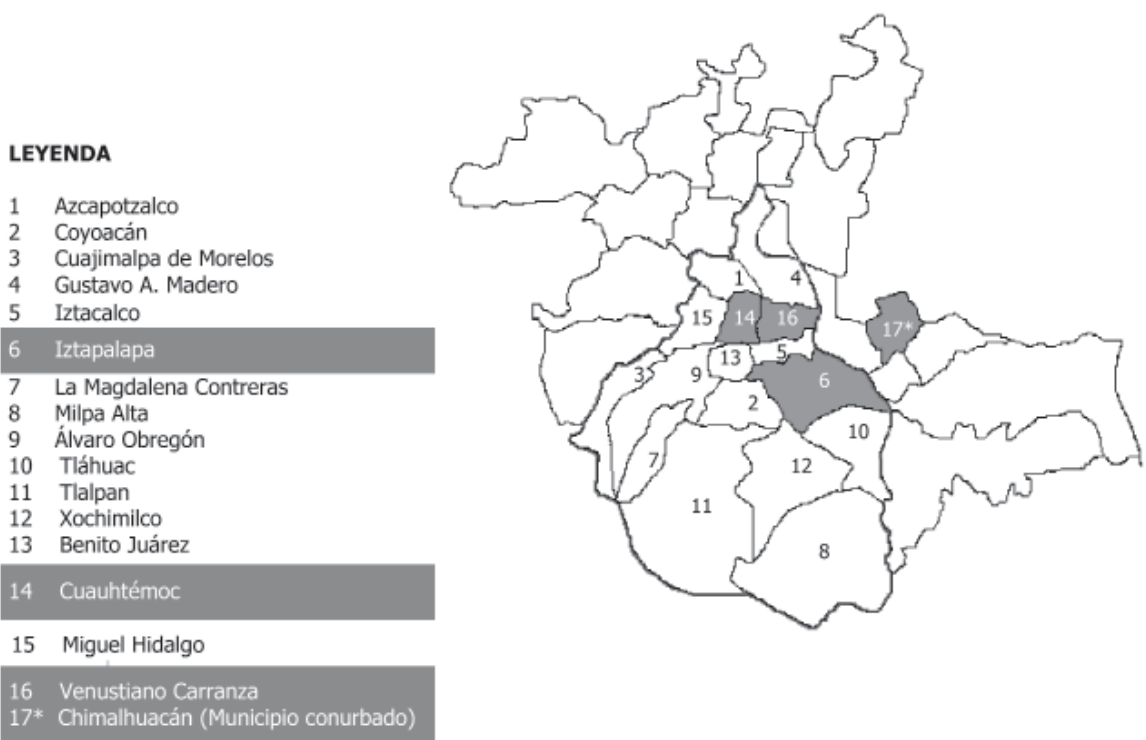

Fuente: Adaptación propia (PUEC-UNAM, 2011)

\section{Ciudad de México. Características y particularidades}

Cuando alguien llega a la Ciudad de México, las siguientes palabras siempre estarán presentes: «México es la ciudad de los superlativos». Una de las ciudades más grandes del mundo, con toda su problemática social, de

7. Por «menores de la calle» se entienden aquellos niños y niñas que habiendo roto el vínculo familiar temporal o permanentemente, duermen en la vía pública y sobreviven realizando actividades marginales dentro de la economía informal callejera, mientras que los y las «menores en la calle», mantienen el vínculo familiar, suelen estudiar y realizan actividades marginales de la economía callejera para sustento propio o para ayudar a su familia. 
infraestructuras, su contaminación, su inseguridad...y también todas sus cosas hermosas, su gente, su arquitectura, su cultura, grande por muchos motivos, y no sólo espacialmente.

Según los datos del Censo General de Población y Vivienda del Instituto Nacional de Estadística, Geografía e Informática de México (INEGI 2000), el Área Metropolitana de Ciudad de México se compone del Distrito Federal (D.F.) con 16 Delegaciones Políticas y de 34 Municipios Conurbados del Estado de México. Los datos del Conteo General de Población y Vivienda (INEGI 2005) arrojan que el Distrito Federal tiene una población de 8.720.916 habitantes, siendo la población total del Área Metropolitana de la Ciudad de México (INEGI 2003) de 17.844 .829 personas, constituyendo la población de 0 a 14 años el 22,5\% de la población total. Sin embargo, datos extra oficiales hablan de una población total cercana a los 24 millones de personas en el Área Metropolitana de la ciudad, siendo estos datos una fuente que ayuda a comprender las complejas dimensiones donde las niñas trabajadoras desarrollan su actividad.

Ciudad de México es una de las ciudades más populosas del mundo, crisol de culturas, de oportunidades y de referentes sociales e históricos. Paseando por sus calles, plazas y avenidas, se encuentra una variada población que ha hecho de la calle su hábitat propio de subsistencia: mercados, tianguis $^{8}$, venta ambulante, puestos improvisados... y son algunas de estas formas de vida, en las que se hace difícil separar el ámbito formal del informal, o el ámbito privado del ámbito público.

Dentro de esa variada gama de gente trabajadora, frecuentemente, se encuentra a niñas en diversas actividades urbanas: ayudando y trabajando en puestos de comidas, verduras y otros artículos, vendiendo en los semáforos, en el metro, en los micros ${ }^{9}$, desarrollando estos trabajos dentro del ámbito familiar o a través de una relación contractual (implícita o explícita).

Las edades son variables así como el desempeño de tareas y la propia percepción y visualización del concepto de trabajo. Según datos que se arrojan sobre el fenómeno del trabajo infantil, hay más niños que niñas trabajando fuera de sus casas, mientras que las niñas son solicitadas para trabajar en las labores domésticas. Pero es precisamente el sector de niñas que trabajan en

8. Tianguis, palabra procedente del náhuatl que significa mercado al aire libre, que puede ser temporal o permanente en determinadas áreas urbanas, aunque inicialmente suelen ser móviles, también llamados a veces mercados sobre ruedas.

9. Los micros son autobuses pequeños, que realizan transporte público desde la iniciativa privada, también son llamados peseros porque antiguamente su precio era de un peso mexicano (en la actualidad el precio oscila entre dos y cuatro pesos). 
las calles y espacios públicos donde radica el interés de este artículo, aquellas niñas que desarrollan sus labores en mercados, vendiendo productos en diferentes espacios de la ciudad, cantantes, músicas, payasitas, pepenadoras ${ }^{10}$, limpiacristales, empaquetadoras en supermercados... una gran variedad que en demasiadas ocasiones no es analizada con toda la seriedad que precisa.

Ciudad de México, por tanto, puede ser considerada como uno de los paradigmas en cuanto a las grandes megalópolis latinoamericanas se refiere. Una populosa ciudad que constituye en sí misma grandes oportunidades y grandes retos de la vida urbana. Multiplicidad de elementos que hacen de ella una ciudad compleja y llena de desafíos, especialmente para las mujeres y las niñas.

\section{La ciudad y las niñas: ¿esferas incompatibles?}

En México las raíces del trabajo infantil hay que buscarlas en diferentes aspectos (UNICEF, 1999): por un lado, están las formas que adoptó el desarrollo, con desequilibrios regionales, desigual distribución del ingreso, crecimiento urbano explosivo, dispersión y aislamiento de la población rural. Este hecho se ve claramente en los fuertes procesos migratorios que sufrió Ciudad de México en diferentes décadas del siglo XX, pero con especial intensidad desde 1980 hasta la actualidad, en la llamada Fase de Megalopolización (Luna y Gómez, 1992). Por otro lado, los nuevos procesos sociales fueron causa de este fenómeno: corrientes migratorias que incorporaron cada vez más a crecientes contingentes de menores y mujeres, provocando la desintegración familiar y el crecimiento de hogares monoparentales (principalmente encabezados por mujeres), que a su vez generaron cambios en las pautas y valores culturales.

La Política económica por su parte, ha fomentado la creación de empresas familiares, que precisan de mano de obra y que se han apoyado en las redes de parentesco para obtenerla. Todas estas circunstancias tienen como consecuencia que una de cada cinco familias no obtenga ingresos suficientes para la alimentación y uno de cada dos habitantes del campo y uno de cada nueve de los que viven en la ciudad se encuentre en condiciones de extrema pobreza (Estrada, 1999b).

Según datos aportados por UNICEF (1999: 12-18) sobre niños, niñas y jóvenes en situación de calle en el Distrito Federal, el número ascendía a $14322^{11}$. Es importante destacar, que según datos del mismo estudio, del total

10. Pepenar es buscar entre las basuras y desechos aquellos artículos, alimenticios o no, que puedan ser revendidos posteriormente (de ahí el concepto de pepenadoras).

11. Sobre el trabajo infantil en México hay una publicación más reciente del INEGI de 2004 (ver referencia completa en la bibliografía), pero no refleja los datos específicos del trabajo infantil en la Ciudad de México de manera desagregada. 
de niños y niñas en situación de calle, el 86\% (11514) eran trabajadores: 7582 varones (66\%) y 3942 mujeres (34\%). La principal actividad que desempeñan en las calles, en todos los grupos de edad, es el comercio callejero (ventas), debido a que ofrece una mejor rentabilidad por hora de esfuerzo, ocupando esta actividad en las niñas el $88,7 \%$ y en los niños el $61,2 \%$, teniendo en cuenta tanto la venta ambulante como la venta en puestos fijos. La siguiente actividad en importancia para los niños es la de limpiacristales y diableros ${ }^{12} \mathrm{y}$ en las niñas, la de payasitas/actrices y actividades de mendicidad.

En todos los espacios donde se realizó el trabajo de campo, se buscaron diferentes tipologías de trabajo infantil, y aunque el objetivo eran las niñas, no podía dejar fuera a los niños, los censos dicen que numéricamente ellos son muchos más, pero no es difícil encontrarlas a ellas. Quizá, salvo determinados trabajos como los diableros, niñas y niños desempeñan funciones similares. No encontré niñas con el diablo, pero tampoco vi a niños de lava trastes, esa tal vez sea la diferencia más evidente, pero en el resto de funciones que se pueden desempeñar, encontré a unos y a otras indistintamente. Al tener vínculos familiares, generalmente los trabajos van relacionados con la actividad profesional que desempeñan los adultos de la unidad doméstica, si la madre y el padre tienen un puesto de fruta, tanto niños como niñas trabajarán en el puesto. Las diferencias de género vienen dispersas en otros factores, no en el desempeño en sí mismo, sino en las formas de pago, en la capacidad de decidir si trabajan o no en otros lugares, o en el uso del tiempo para disfrutar de las amigas o los amigos.

Ser niña y ser mujer en México (al igual que ocurre en muchos otros lugares del mundo) tiene una carga moral compleja, ya que el mercado y las calles donde trabajan son un espacio común, donde todo se sabe, y ellas deben cuidar su imagen, se debe mirar por dónde andan y con quién. Los niños son considerados, al fin y al cabo, por su propia «naturaleza» como más rebeldes, más independientes y autónomos...volviendo a caer en las dicotomías del modelo androcéntrico, y por consiguiente, el nivel de exigencia y control hacia ellos es menor.

Pues, usted bien sabe que una mujer es más peligroso que a un hombre y la verdad pues sí, a las mujeres les digo: "Tal hora» y a tal hora tienen que estar aquí, las muchachas me preocupan más que los hombres (Madre de niña trabajadora de 15 años).

En los permisos, yo siento que a él (su hermano) le dan más permisos por lo mismo que es hombre, pero sí, cuando yo quiero salir, nada más me dice mi mamá

12. Carretilleros, en relación a que cargan los diablos (carretillas). 
«con quién vas, y te quiero aquí a una hora», o ella me espera hasta que llegue y todo (Niña trabajadora. 14 años).

La presencia femenina depende de los entornos y de los diversos trabajos que se realicen en el mismo, tal y como se puede ver en ámbitos de cocinas, comidas o venta de productos alimenticios, en los que la visibilidad es mayor debido a la extensión del rol «doméstico» de las mujeres realizado en el ámbito público, tal y como comentaban cuando preguntaba en qué trabajan mujeres y hombres y niños y niñas. En la mayoría de las ocasiones, respondían que las mujeres trabajan en comidas y alimentación y que los hombres no (cuando en la realidad también se ve a muchos hombres que trabajan en alimentación) o también haciendo referencia a que las niñas trabajan cuidando de hermanos y hermanas menores mientras sus hermanos varones trabajan fuera de casa:

Las mujeres trabajan en las cocinas, hacen de comer, van por tortillas, lavan trastes (...) hombres casi no hay...bueno, sí hay pero trabajan en cosas diferentes como la tortillería, venden llaves, café (Hermano de niña trabajadora. 13 años).

Otras niñas también se quedan cuidando a sus hermanitos mientras los hermanos trabajan fuera (Niña trabajadora. 9 años).

En otros ámbitos, vemos cómo el discurso y la práctica caen continuamente en contradicciones, ya que las mujeres reconocen mayoritariamente que trabajan más horas que los hombres y eso se muestra en los ingresos económicos, pero aún sobrevuela la imagen de que el hombre debe asumir «el gasto» de la familia. Aún se tiene la imagen del hombre como «cabeza de familia», y es interesante en los hogares encabezados por mujeres ver cómo la ausencia de estos hace que ellas se vuelvan más estrictas a la hora de controlar a sus hijos y sobre todo a sus hijas, ya que hay cierto miedo al acoso por «aparentar» vulnerabilidad, sin una figura masculina que las «proteja». Esa vulnerabilidad que se presume de las niñas, se va asimilando por parte de ellas y se traduce en una mayor dependencia hacia los hombres (que pueden ser sus padres, su hermanos o amigos, compañeros o novios) haciendo además que desarrollen peores expectativas de futuro, muchas de ellas ni siquiera se han planteado el futuro, o este se sigue orientando a trabajos de atención y cuidado

\subsection{Uso del espacio urbano. Desdibujando la dicotomía privado-público}

Dentro de las muchas cuestiones que se pueden analizar en torno al trabajo que realizan niñas y mujeres en la ciudad encontramos especialmente que, tal y como el imaginario colectivo nos recuerda, se trata de una ciudad llena de «amenazas». Es un espacio masculinizado, en el que las mujeres están limitadas en sus movimientos y oportunidades, pero que incide de manera mucho 
más virulenta si sobre la discriminación por género, añadimos otros factores como la discriminación por estatus socio-económico, por la edad o la etnia. Podríamos concluir por tanto, que es un lugar «inviable» para las niñas y «poco recomendable» para las mujeres.

Sin embargo, es preciso matizar y analizar cómo se construyen los discursos (en los que se suele caer en la demagogia simple de «vulnerabilizar» todas las cuestiones que atañen a mujeres y niñas), y debemos revisar cómo sobreviven ellas a los espacios, demostrando cotidianamente sus muchas potencialidades a pesar de las adversidades que el entorno les ofrece.

\subsubsection{La Casa}

En primer lugar, es preciso revisar el concepto de «la casa» (ámbito privado) como lugar «seguro» para las niñas trabajadoras urbanas. Según la mayoría de los discursos y experiencias analizadas, el uso del espacio de «la casa» propiamente dicho, es relevado a un segundo puesto en gran parte de ocasiones, o al menos, está a un mismo nivel de importancia que el que puede suponer el lugar de trabajo. Debemos tener en cuenta, que la casa, para ser un espacio de caracterización y pertenencia debe tener, por un lado, condiciones adecuadas de habitabilidad, hecho que en muchos casos no se da debido a las precarias condiciones económicas (casas de láminas y cartón, con suelo de tierra, predios ocupados, cuartos en vecindades con baños y lavaderos en el exterior...). Además, debido a las grandes distancias de la ciudad, pasar tiempo en la casa se torna difícil por las intensas jornadas laborales y vitales. Tal y como contaban estas niñas:

De ahí por donde vivo no le hablo a casi nadie, porque nada más salgo de mi casa, me vengo al mercado, regreso a mi casa a las diez de la noche y ya no salgo de ahi (Niña trabajadora. 14 años).

No, aquí (en el lugar de trabajo) es todo el día, siempre estoy aquí, la casa nada más la utilizamos para ir a dormir (Niña trabajadora. 15 años).

Vemos, por tanto, que la casa, está en segundo plano en cuanto al uso e identificación, produciéndose paralelamente en los espacios laborales, una extensión del uso que va más allá de lo que se suele considerar tarea productiva, produciéndose una domesticación del espacio público. Vemos que tanto la casa como la calle (referida al espacio laboral concreto) tienen una dimensión pública y una dimensión doméstica aunque con significados y usos simbólicos diferentes. Por un lado, la calle (en cualquiera de las formas de trabajo que en ella se desarrollan) tiene la dimensión pública al ser considerada el lugar generador de ingresos y como lugar de socialización (donde los códigos condicionan los comportamientos y donde se tienen en cuenta las jerarquías de 
género y edad) y tiene una dimensión doméstica en el uso de sus espacios como prolongación del hogar (con comportamientos que inicialmente se darían dentro del ámbito privado); por otro lado, la consideración de la casa tiene una dimensión pública en cuanto a que hace referencia a un espacio que va más allá de las "cuatro paredes» con el uso de espacios compartidos como los lavaderos o los baños (debido a las condiciones de las viviendas, que en muchos casos son pequeños cuartos con espacios comunes, como son los llamados terrenos o las vecindades) o incluso las calles aledañas a la vivienda y una dimensión doméstica que se refiere a los aspectos más íntimos y privados.

La mayoría de amistades son de aquí del mercado, todo el día se la pasan aquí, también de la escuela... pero más, más convive con los del mercado, básicamente no está uno en la casa (Madre de niña trabajadora de 9 años).

\subsubsection{La Calle}

Revisando teóricamente el concepto de «la calle» (Falú, Morey y Rainero, 2002: 40) vemos que esta es el espacio de los hombres, para quienes las relaciones sociales están centradas en diferentes actividades según el contexto y la realidad (pudiendo ser espacios violentos para las mujeres y las niñas). La situación de inseguridad urbana en las ciudades, producto del deterioro socio-económico, alcanza situaciones críticas e impacta particularmente en la vida de las mujeres y las niñas y sus posibilidades de recreación barrial, caracterizada en general por condiciones de hacinamiento. El espacio físico constituye, sin duda, una dimensión activa que condiciona las formas de uso y las interacciones entre las personas. Las actividades ocurren en el espacio y este, a su vez, dependiendo de las características y calidad, posibilita o reafirma un determinado comportamiento.

Pero debemos considerar también que para mujeres y niñas, la posibilidad de salir al ámbito público (aún las que realizan trabajos de baja calificación) les permite generar vínculos y relaciones que en muchos casos son significativos para sus experiencias de vida. Para Alejandra Massolo (1992) hay una «inmensa laguna de ignorancia» sobre la dimensión de género en las investigaciones urbanas, sin ser esta dimensión considerada en la lista de «ausencias» de aspectos que han sido descuidados y que afectan al mejor conocimiento y explicación de las problemáticas de las ciudades.

A su vez, la calle (González, 1999: 547-548), se nos presenta como el espacio donde se ponen en marcha los aprendizajes adquiridos en la casa y en la escuela, es el lugar donde se llevan a cabo las reglas del juego de la propia vida. La calle viene a ser la arena de la puesta en marcha de las disposiciones culturales aprendidas, se presenta como el lugar de trabajo, de comercio y de 
recreación, como el lugar de las prácticas sociales, de los desplazamientos y movimientos. La calle es un espacio de múltiples interacciones y polisémico, presentándose a veces con una doble textura, una doble característica que se configura al mismo tiempo como lo propio y lo ajeno. Esta doble vivencia experimentada en la calle (lo que podría definirse como una suerte de espacio público dual) origina que se incorporen al aprendizaje diversas y variadas disposiciones culturales que provienen de dos campos socioculturales: el externo y el interno.

Para Manar Hammad (en Sonesson, 2004: 149), cuando hablamos de un espacio público, no sólo tendremos que analizar su función, sino cuáles son las potencialidades para la acción que este nos abre. Por este motivo, resulta interesante mostrar aquí cuál es el uso y la apropiación del espacio laboral y del espacio vital que realizan las mujeres y niñas mexicanas, para evitar caer en distorsiones conceptuales que luego tendrán repercusión en los programas de atención social que se generan en torno a ellas.

A lo largo de la estancia en la ciudad, observé cómo la gente trabajadora se va apropiando de los lugares donde pasan la mayor parte de su tiempo, y a pesar de que algunos inicios del trabajo en la calle pueden ser duros, la costumbre y el entorno se configuran como algo propio que les hace sentirse bien. La calle se convierte en un segundo espacio de socialización fuera del entorno familiar no como espacio neutro y asexuado, sino contemplado en toda su dimensión y magnitud, mostrando múltiples elementos con los que las mujeres y niñas trabajadoras se van conformando e identificando. Tal y como contaban estas niñas:

Pregunta: ¿y qué es lo que más te gusta de lo que haces? Respuesta: de que estoy en la calle (Niña trabajadora. 11 años).

Al principio era muy, muy pesado, porque yo todavía no sabía el movimiento de las personas, que te empiezan a decir de cosas, y después les contestaba, pero ya a casi a todos les hablo, me hice muchos amigos (Niña trabajadora, sobre su incorporación al trabajo en uno de los grandes mercados de la ciudad. 15 años).

La oposición entre lo privado y lo público queda desdibujada por los usos y los discursos recogidos a través de la etnografía realizada. Esa domesticación de lo público, quizá no se llegue siempre a reconocer explícitamente en los discursos, pero en el tiempo de observación en la calle vi cómo la gente duerme en los lugares más insospechados (detrás de los mostradores de los locales, en un par de sillas superpuestas, en un diablo tumbado en el suelo), cómo las niñas y niños hacen los deberes escolares entre pucheros de cocina, rodeados de ruidos o música o a la vez que trabajan en las ventas de un puesto, cómo se cuidan los bebés (con cajas improvisadas como cunas), dónde comen (en 
los puestos de comidas de «confianza» de los propios mercados, en el mismo espacio de las ventas donde te sirven «a domicilio» la comida caliente, o el suelo de la zona donde siempre están, que ya no es un suelo cualquiera, sino que tiene cierto sentido de propiedad), dónde se peinan o lavan los dientes, dónde ven la televisión o escuchan música o dónde hacen las cuentas de los gastos...todas ellas, actividades más «propias de hacer en casa».

\subsection{Sentido de pertenencia y percepción del peligro}

Los espacios laborales donde trabajan las niñas y mujeres, a lo largo del año y en función de la temporada que se acerque (Navidades, Día de la Independencia, Carnavales...), se van tornando de colores variados, con estructuras diferentes...recreándose y reinventándose continuamente, mostrando lo vivos que están y lo mucho que pueden llegar a sorprender a quien pase por allí.

En una gran cantidad de ocasiones, el trabajo que realizan las niñas está dentro de la red familiar y las tareas que desempeñan forman parte de una tradición que les hace sentirse en su lugar de trabajo como parte de él, es decir, pertenecen y se identifican a ese lugar. Sobre este sentido de pertenencia, podemos ver cómo este cambia según la edad de las personas, el tiempo que lleven allí, y las dinámicas en las que estén envueltas, pero sin duda, su lugar de trabajo forma parte de su identidad y socialización.

Ellos (los trabajadores del mercado), me creen como su hermana, y así, ahorita, antes te decían algo y nadie te defendía, ahorita todos me defienden, si una persona te dice algo ellos van y les dicen, que se calme y ya (Niña trabajadora. 13 años).

A pesar de las múltiples adaptaciones, es innegable que los entornos laborales son complejos, destacando de estos, especialmente, cuatro aspectos comunes y que son determinantes para el desempeño de las tareas cotidianas: el ruido, la basura, la inseguridad y el acoso, y que son aspectos que suponen o pueden suponer un peligro para su integridad física o psicológica.

La percepción del peligro, sin embargo, debe ser contextualizada, para evitar así, conclusiones deterministas que son utilizadas posteriormente para posicionamientos y programas políticos y sociales que poco tienen que ver con la realidad que les acompaña a ellas. Aún así, no siempre el peligro es percibido de manera amenazante debido a que ellas son "parte del lugar» y en todo caso, supone un peligro para la gente de «fuera», estructurándose toda la red social y de parentesco en torno a la gente trabajadora y siendo esto un factor de protección invisible desde el exterior, pero que resulta altamente eficiente y tiene una gran cantidad de símbolos y prácticas que disminuyen los riesgos y establecen otros elementos de protección. 
A mí esta zona no se me hace peligrosa, siempre y cuando no provoques a la gente, porque cuando la provocas, encuentras, pero a mi parecer no es peligroso. Peligroso Tepito, Morelos, ahí si te digo que es peligroso, pero la Merced, no. (Niña trabajadora. 10 años).

Yo creo que siempre ha tenido fama de barrio bravo pero no creo que.... a veces es más su mala reputación que lo que realmente es cierto, porque bueno, depende, ¿verdad? Si yo paso a las 10, 11 de la noche, no me hacen nada, porque me conocen, pero si otra persona que no es de aquí pasa, obviamente que sí (Niña trabajadora. 15 años).

De igual modo, tal y como hemos visto, la percepción del peligro está asociada a las niñas o a las mujeres debido a su condición (según los estereotipos de género que les atribuyen debilidad, fragilidad y vulnerabilidad frente al hombre), hecho que nos indica que todavía las libertades, el uso de los espacios y el nivel de autonomía para ellas se encuentra limitado, y por ello deberemos de trabajar, cambiando esos factores discriminatorios que poco ayudan a un ejercicio libre de sus derechos.

Llego a la casa como a las 7:00, llego a calentar mi agua para bañarme y ya después, antes de bañarme salgo a jugar con mis amigos y ya después me meto a bañar, comemos...(...)los chicos andamos así en cualquier lado...con los que me junto andamos jugando y eso, y nos cuidamos, entonces ella (refiriéndose a su hermana) que es mujer no la dejan, pues un hombre se puede defender y una mujer, aunque se quiera defender, pues no es lo mismo, siempre un hombre va a tener la de ganar (Hermano de niña trabajadora. 17 años).

Ella (la niña) se queda con su papá vendiendo y él (el niño) se va solo, una mujer es más peligroso para andar solita en la calle, un chamaco no es igual que una mujer, si él se quiere ir a trabajar que se vaya solito, pero su papá dice: «aquí lo que manda soy yo, y si quieren irse ellas que se vayan con su papá» (Madre de niña trabajadora de 11 años).

P: ¿Quién lava la ropa? R: Mi mamá y nosotras que somos mujeres...lavamos otras cosas de los grandes, cada quien de nosotras lava nuestra ropa, la que hay; y ya mi mamá lava, nosotras un poquito de cada uno de mis hermanos, y ya mi mamá lava la de mis hermanitos chiquitos y un poquito de las chamarras (chaquetas, abrigos) P: ¿los hombres lavan ropa? R: No P: ¿Y quién cocina? R: Mi mamá y nosotras le ayudamos (Niña trabajadora. 10 años).

\section{Conclusiones...o cómo deconstruir esas barreras}

A través de estas páginas se ha tratado de mostrar un enfoque que sirva de punto de partida para el análisis y consideración teórica sobre el trabajo infantil, que es preciso ir «resignificando» evitando así determinismos y categorizaciones vacuas que no cuestionan las estructuras y jerarquías de dominación que durante mucho tiempo han sobrevolado a la consideración de la infancia 
y que especialmente imaginan a las niñas como «sujetos susceptibles de intervención» en mayor medida que a los niños, sin valorar otros elementos condicionantes de la inequidad.

Es cierto que en todos los espacios laborales analizados (mercados públicos, tianguis, basureros, plazas públicas), de una manera u otra, se dan situaciones de violencia, prostitución, suciedad o acoso, pero tanto en los discursos como en las prácticas se da una relativización y normalización del peligro, porque es con lo que les ha tocado vivir. Debemos considerar que en el fondo, toda la ciudad es «peligrosa» tanto para hombres como para mujeres (nadie está exento de que le ocurra algo) y que dependiendo de cómo percibimos ese peligro, nos condicionará más o menos para realizar determinadas actividades. Sin embargo, las niñas demuestran, día a día, que son capaces de transformar sus entornos haciéndolos más cercanos, apropiándose de ellos y desmitificando los prejuicios, porque la vida debe continuar.

Es fundamental trabajar por mejorar las condiciones vitales de los entornos laborales urbanos, no sólo de las mujeres y las niñas, sino de todas las personas, evitando enfoques "proteccionistas» que subrepticiamente conlleven privación de libertades y acceso limitado para las mujeres (desde su infancia a su madurez). Ellas son capaces de adaptarse y modificar su entorno y es preciso escuchar sus voces antes de tomar iniciativas que hagan cada vez más grande la brecha de género. Habrá que establecer programas que aboguen por la participación infantil, por los derechos humanos, y no programas que predispongan al colectivo de mujeres y niñas en situación de desventaja social, incapacitándolas y mermando sus potencialidades y anulando sus posibilidades de empoderamiento individual y colectivo. Habrá también que plantear un enfoque que trabaje por eliminar las desigualdades por encima de un enfoque que plantee la vulnerabilidad como punto de partida.

Los estados y las políticas públicas deben ser un eje vertebrador de protección y provisión, pero también establecer canales de participación y el planteamiento hacia las niñas debe ser de promoción y valoración, al menos, en igualdad de condiciones que el resto de la sociedad.

La propuesta, por tanto, es seguir construyendo ciudadanía sin caer en dicotomías excluyentes, valorando capacidades y analizando la realidad en toda su complejidad, cualitativa y progresivamente. Sólo así contribuiremos a una sociedad más justa y equitativa y tendremos unas ciudades mucho más habitables para todas y todos. 


\section{Bibliografía}

Aitken, S., Estrada, S., Jennings, J., Aguirre, L. M. (2006). Reproducing Life And Labor: Global processes and working children in Tijuana, Mexico. Childhood, 13 (3).

AlarCon, W. (1994). Ser niño. Una nueva mirada de la infancia en el Perú. Lima: UNICEF-IEP.

Beauvoir, S. (1952). The Second Sex. New York: Vintage Books.

Bolton, A., Pole, C., Mizen, P. (2001). Picture This: Researching Child Workers. Sociology 35, 2, 501-518.

BONTE, P., IZARD, M. (1996). Diccionario de Etnología y Antropología. Madrid: Akal.

CArrasco, C. (1996). Presente y futuro del trabajo. Apuntes para una distribución no androcéntrica. En A. Rodríguez, B. Goñi y G. Maguregi (Eds.). El futuro del trabajo. Reorganizar y repartir desde la perspectiva de las mujeres. Bilbao: Bakeaz-CDEM.

Carrasco, C., Mayordomo, M., Domínguez, M. y Alabart, A. (2004). Trabajo con Mirada de Mujer. Propuesta de una encuesta de población activa no androcéntrica. Madrid: Consejo Económico y Social. Colección Estudios. № 155.

Diallo, Y., Hagemann, F., Etienne, A., Gurbuzer, Y., Mehran, F. (2011). Evolución mundial del trabajo infantil: Evaluación de las tendencias entre 2004 y 2008. Ginebra: OIT-IPEC.

Dorman, P. (2001). Child Labour in the Developed Economies. Geneva: International Labour Office, International Programme on the Elimination of Child Labour.

ESTRADA, M. (1999a). Infancia y trabajo. La experiencia de los sectores populares urbanos. Estudios Sociológicos, XVII: 49, 54-73.

Estrada, M. (Coord.). (1999b). Familias en la crisis. México: CIESAS-SEP-CONACYT.

Falú, A., Morey, P., Rainero, L. (2002). Ciudad y Vida Cotidiana. Asimetrías en el uso del tiempo y del espacio. Argentina: Red Mujer y Hábitat de América Latina.

Friedan, B. (1963). The Feminine Mystique. New York: Norton.

Gaitán, L., Martínez, M. (2006). El enfoque de derechos de la Infancia en la Programación. Guía para el diseño, desarrollo y evaluación de proyectos. Monografías del Experto en Políticas Sociales de Infancia. Serie Práctica No 1 . Madrid: UCM.

GONZÁlez, F. (1999). La casa, la calle y la escuela como instancias de socialización en dos comunidades étnicas mazahuas del Estado de México. En A. Civera (Coord.). Experiencias educativas en el Estado de México. Un recorrido histórico. México: El Colegio Mexiquense. 
Guerra, E., CAlderón, G., Soto, B. (2007). Consideraciones metodológicas en el estudio del trabajo infantil. Gaceta Ide@s, CONCYTEG, 2, 26.

Hilowitz, J. (2004). Trabajo Infantil: Un manual para estudiantes. Ginebra: OIT.

Hungerland, B., Liebel, M., Milne, B., Wihstutz, A. (Eds.). (2007). Working to Be Someone. Child Focused Research and Practice with Working Children. London/Philadelphia: Jessica Kingsley Publishers.

INEGI (2000). XII Censo General de Población y Vivienda. México: INEGI.

INEGI (2003). Cuaderno Estadístico de la Zona Metropolitana de la Ciudad de México. Estado y Movimiento de la Población. México: INEGI.

INEGI (2004). El Trabajo Infantil en México. 1995-2002. México: INEGI.

INEGI (2005). II Conteo de Población y Vivienda. México: INEGI.

Leyra, B. (2009). Trabajo Infantil Femenino. Niñas trabajadoras en Ciudad de México. Colección Digital de Tesis Doctorales de la Universidad Complutense de Madrid. ISBN: 978-84-692-6017-3. Disponible en: http://eprints.ucm. es/9394/1/T31016.pdf

LIEBEL, M. (2003). Infancia y Trabajo. Lima: IFEJANT.

Liebel, M. (2006). Entre Protección y Emancipación. Derechos de la Infancia y Políticas Sociales. Monografías del Experto en Políticas Sociales de Infancia. Serie Teoría No 1. Madrid: UCM.

López-CALVA, L. F. (2007). Trabajo infantil: teoría y lecciones de la América Latina. México: Fondo de Cultura Económica.

LunA, M. A., GómEZ, R. (1992). Límites al crecimiento de la Zona Metropolitana de Ciudad de México. En VV.AA. La zona metropolitana de la ciudad de México. Problemática actual y perspectivas demográficas y urbanas. México: CONAPO.

MARTIN, K., VoORHIES, B. (1978). La Mujer: Un enfoque antropológico. Barcelona: Anagrama.

Massolo, A. (Comp.). (1992). Mujeres y Ciudades. México: PIEM-Colegio de México.

ORTNER, S. (1979). ¿Es la mujer con respecto al hombre lo que la naturaleza con respecto a la cultura? En O. Harris, y K. Young (Coords.). Antropología y feminismo. Madrid: Anagrama.

Pateman, C. (1988). The Sexual Contract. Palo Alto, CA: Stanford University Press.

PUEC-UNAM (2011). Propuestas para el desarrollo económico, social y urbano en el Distrito Federal. México: Programa Universitario de Estudios de la CiudadUniversidad Nacional Autónoma de México.

Rosaldo, M. (1979). Mujer, cultura y sociedad: una visión teórica. En O. Harris, y K. Young (Coords.). Antropología y feminismo. Madrid: Anagrama.

SONESSON, G. (2004). Espacios de urbanidad. De la plaza aldeana al bulevar. POTLATCH. Cuaderno de Antropología y Semiótica. Año 1, 1, 23-45. 
UNICEF (1997). Estado Mundial de la Infancia. Tema: Trabajo Infantil. Nueva York: UNICEF.

UNICEF (1999). Informe número 11 sobre Trabajo Infantil. México: UNICEF.

Wollstonecraft, M. (2008). A Vindication of the Rights of Women. New York: Cosimo.

WoodheAd, M. (2004). Psychosocial Impacts of Child Work: A Framework for Research, Monitoring and Intervention. International Journal of Children's Rights 12, 4, 321-377. 